J. Phys. IV France 127 (2005) 53-61

(C) EDP Sciences, Les Ulis

DOI: $10.1051 /$ jp4:2005127009

\title{
Microscopie par rayons X dans la fenêtre de l'eau : faisabilité et intérêt pour la biologie d'un instrument de laboratoire
}

\author{
J.F. Adam ${ }^{1}$ et J.P. Moy ${ }^{1}$ \\ ${ }^{1}$ Département des Micro-Technologies pour la Biologie et la Santé, LETI-CEA, \\ 17 rue des Martyrs, 38054 Grenoble Cedex 9, France \\ e-mail : jean-pierre.moy@cea.fr
}

\begin{abstract}
Résumé. La biologie étudie des structures ou des phénomènes sub-cellulaires. Pour cela la microscopie est la technique d'observation privilégiée. La résolution spatiale de la microscopie optique s'avère bien souvent insuffisante pour de telles observations. Les techniques plus résolvantes, comme la microscopie électronique par transmission sont souvent destructrices et d'une complexité peu adaptée aux besoins des biologistes. La microscopie par rayons $\mathrm{X}$ dans la fenêtre de l'eau permet l'imagerie rapide de cellules dans leur milieu naturel, nécessite peu de préparation et offre des résolutions de quelques dizaines de nanomètres. De plus, il existe un bon contraste naturel entre les structures carbonées (protéines, lipides) et l'eau. Actuellement cette technique est limitée aux centres de rayonnement synchrotron, ce qui impose une planification et des déplacements incompatibles avec les besoins de la biologie. Un tel microscope fonctionnant avec uns source de laboratoire serait d'une grande utilité. Ce document présente un état de l'art de la microscopie par rayons $\mathrm{X}$ dans la fenêtre de l'eau. Un cahier des charges détaillé pour un appareil de laboratoire ayant les performances optiques requises par les biologistes est présenté et confronté aux microscopes $\mathrm{X}$ de laboratoire déjà existants. Des solutions concernant la source et les optiques sont également discutées.
\end{abstract}

\section{INTRODUCTION}

La compréhension des mécanismes mis en œuvre au niveau cellulaire et sub-cellulaire est cruciale pour l'étude des organismes vivants, qu'ils soient sains ou pathologiques. L'analyse cellulaire morphologique ou fonctionnelle de cellules cancéreuses permet notamment de guider certains choix thérapeutiques et d'améliorer leur efficacité. Les techniques d'imagerie de la cellule, dont la microscopie représente la composante principale, jouent un rôle fondamental dans cette compréhension. Ainsi, les nouvelles techniques de microscopie ont toujours ouvert de nouvelles perspectives scientifiques. Cela a déjà été le cas avec les premiers instruments optiques fabriqués par Anton van Leeuwenhoek [1] et est toujours vrai pour les techniques plus récentes telles que la microscopie électronique à transmission [2] ou la microscopie optique à champ proche [3]. En contrepartie, le développement de nouveaux instruments est souvent motivé par la demande scientifique. Ainsi les prouesses technologiques des physiciens dans la réalisation de nouveaux outils d'imagerie doivent répondre aux besoins réels des biologistes.

\subsection{Les besoins de la biologie cellulaire}

Les besoins et les contraintes spécifiques de la biologie cellulaire en termes d'outil d'imagerie microscopique permettent d'imaginer quelles doivent être les performances de l'instrument idéal.

Le paramètre fondamental est la résolution spatiale. Le brin d'ADN fait environ $2 \mathrm{~nm}$ de diamètre, il est enroulé en fibres de nucléosomes de $10 \mathrm{~nm}$ de diamètre qui elles mêmes sont enroulées en superstructures (fibres de chromatine) de $30 \mathrm{~nm}$ de diamètre [4]. Le chromosome qui est la structure finale, a un diamètre de 1 à $2 \mu \mathrm{m}$. Une mitochondrie a un diamètre de 0.5 à $1 \mu \mathrm{m}$ pour une longueur de 5 à $7 \mu \mathrm{m}$. Les structures internes de ces mitochondries et les vésicules lipidiques ont un diamètre de l'ordre de quelques dizaines de nanomètres. Ces exemples donnent l'échelle d'intérêt en biologie cellulaire, et expliquent qu'un 
instrument ne permettant pas de voir ces structures n'offrirait pas de réel progrès face aux technologies existantes.

Un autre point clé est de pouvoir faire de l'imagerie de cellules adhérentes. En effet, l'adhérence cellulaire est à la base de l'organisation tissulaire des cellules [5], et la plupart des cellules ont besoin d'un support pour se développer. De plus, l'observation de cellules entières (3D), si possible vivantes (au minimum dans leur milieu naturel), est essentielle pour un repérage précis des structures étudiées.

La disponibilité de marqueurs est aussi un paramètre capital, car on veut voir des phénomènes moléculaires et les localiser dans la cellule. Il faut donc disposer d'agents de contraste spécifiques. Pour cela, une approche multimodale est souvent nécessaire car une technique permettra de mettre en évidence le phénomène recherché, alors qu'une autre permettra de le localiser [6]. Dans les laboratoires de biologie les techniques d'imagerie permettant cette approche multimodale sont disponibles à tout moment.

\subsection{Les techniques d'observation actuelles}

\subsubsection{La microscopie optique conventionnelle [7]}

Cette technique est la plus ancienne, et comprend de nombreuses variantes: l'échantillon est éclairé en réflexion ou en transmission. Les molécules à observer vont interagir avec la lumière de plusieurs façons :

- soit en absorbant certaines longueurs d'ondes car les structures à observer sont colorées naturellement ou artificiellement. C'est la microscopie en lumière directe.

- soit en provoquant un déphasage. C'est la microscopie en contraste de phase.

- soit en émettant de la lumière à une autre longueur d'onde que celle d'origine. C'est la microscopie de fluorescence. Il est possible d'utiliser simultanément plusieurs marqueurs qui seront excités par la même lumière, et émettront à des longueurs d'ondes différentes.

La microscopie optique permet une observation morphologique de cellule vivante dans leur milieu d'origine sans préparation par contraste de phase, ou avec une coloration en contratse d'intensité, alors que la fluorescence permet donne une information fonctionnelle.

La résolution spatiale obtenue est cependant insuffisante. Elle est limitée par la diffraction et sa limite inférieure est donnée par l'équation suivante :

$$
R_{L}=\frac{1.22 \lambda}{2 N A}
$$

avec $\mathrm{R}_{\mathrm{L}}$ la résolution limite ; $\lambda$ la longueur d 'onde du rayonnement d'éclairage et NA l'ouverture numérique de l'objectif (NA $<1.3$ dans l'eau). On a donc $\mathrm{R}_{\mathrm{L}}>0.25 \mu \mathrm{m}$ pour un éclairage à $0.5 \mu \mathrm{m}$.

\subsubsection{La microscopie confocale de fluorescence [8]}

Dans cette technique récente, l'échantillon est éclairé à travers l'objectif par un laser introduit grâce à une lame dichroïque, et focalisé sur une zone dont la dimension est approximativement celle de sa tache de diffraction. Grâce àun trou (pinhole) superposé à l'image de la zone focale et placé devant le détecteur, seuls les rayons issus de la zone focale peuvent atteindre le détecteur : la lumière diffusée ou la fluorescence des régions autres que le volume focal, qui brouillent l'image en microscopie conventionnelle sont ainsi bloquées. Il reste alors à balayer l'échantillon en trois dimensions pour en obtenir une carte tridimensionnelle avec une résolution égale au volume focal, soit environ $0.2 \times$ $0.2 \times 1 \mu \mathrm{m}^{3}$ avec les meilleurs objectifs. Des cellules vivantes dans leur milieu naturel peuvent être observées.

Des objets bien plus petits que la résolution sont détectés, puisqu'on visualise par émission des phénomènes ayant lieu au niveau moléculaire, mais leur localisation n'est pas meilleure que la résolution.

Grâce aux développements de la biochimie, une immense variété de marqueurs spécifiques est maintenant disponible. Il est classique d'utiliser plusieurs marqueurs à la fois. 


\subsubsection{La microscopie électronique à transmission [2]}

Cette technique est communément utilisée malgré l'extrême difficulté de préparation des échantillons. En effet, elle offre une résolution spatiale quasi moléculaire, de l'ordre du nanomètre. Le contraste provient des différences d'absorption du faisceau incident d'électrons. Les électrons devant se propager dans le vide, les échantillons sont impérativement secs, fixés chimiquement ou congelés. Ils sont ensuite découpés en tranches fines de $100 \mathrm{~nm}$ d'épaisseur environ, puis colorés à l'aide d'éléments lourds absorbant les électrons. Cette coloration est sujette à controverse car à l'origine de nombreux artefacts dans les images.

Cette technique ne permet donc pas l'imagerie de cellules vivantes dans leur milieu naturel. Il existe également quelques anticorps marqués à l'or colloïdal pour localiser certains phénomènes moléculaires, mais bien moins développés que pour la fluorescence. L'information 3D est obtenue par une opération délicate d'imagerie et de recalage de tranches successives. La tomographie électronique sur des échantillons minces avec des électrons de $300 \mathrm{keV}$ ou plus est en plein développement.

\subsubsection{Les autres techniques de microscopie}

Il convient ici de citer d'autres techniques de microscopie, prometteuses de par la résolution spatiale qu'elles permettent d'atteindre. La microscopie à force atomique (Atomic Force Microscopy, AFM) [9] et la microscopie optique à champ proche (Scanning Near Field Optical Microscopy, SNOM) [3] sont des techniques complémentaires d'analyse surfacique. L'AFM permet d'obtenir la topologie de surface de cellules avec une résolution nanométrique, alors que la SNOM permet d'obtenir une information sur l'absorption (quantité de colorant) avec une résolution de l'ordre de $60 \mathrm{~nm}$. Malgré la simplicité de préparation des échantillons, ces techniques ne permettent pas l'imagerie de cellules vivantes et l'information reste surfacique, sans espoir d'imagerie volumique.

Deux autres techniques de microscopie prometteuses tentent de franchir les limites de la diffraction:

- La microscopie $4 \pi$, amélioration de la microscopie confocale maintenant commercialisée, qui fournit une résolution axiale de $100 \mathrm{~nm}$ sur des cellules vivantes.

- La microscopie à déplétion par émission stimulée (Stimulated Emission Depletion Microscopy, STED), en plein développement, qui offre une résolution latérale de $30 \mathrm{~nm}$ sur des molécules test. Il n'est cependant pas garanti que cette technique prometteuse puisse être utilisée sur des cellules vivantes dans leur milieu. Les marqueurs spécifiques à cette technique restent à développer.

\subsection{L'apport de la microscopie par rayons $\mathrm{X}$ dans la fenêtre de l'eau}

La microscopie par rayons $\mathrm{X}$ dans la fenêtre de l'eau offre des possibilités intermédiaires entre celles de la microscopie optique et celles de la microscopie électronique [11]. La fenêtre de l'eau est la zone d'énergie des rayons $\mathrm{X}$ mous située entre le seuil $\mathrm{K}$ du carbone $(284 \mathrm{eV}, 4.4 \mathrm{~nm})$ et le seuil $\mathrm{K}$ de l'oxygène $(543 \mathrm{eV}, 2.3 \mathrm{~nm})$. Dans cette gamme d'énergie, les matériaux organiques, dont le carbone est l'élément prépondérant (protéines, lipides,...), sont 10 à 20 fois plus absorbants que l'eau (figure 1). De plus la pénétration du rayonnement dans l'eau est de l'ordre de $10 \mu \mathrm{m}$ à $500 \mathrm{eV}$. Les longueurs d'ondes étant 100 fois plus petites qu'en optique, la résolution ultime est de l'ordre du nanomètre.

Avec des rayons $\mathrm{X}$ dans la fenêtre de l'eau, il est donc possible de faire de la microscopie haute résolution plein champ, en transmission, de cellules entières dans leur milieu naturel, et présentant un bon contraste intrinsèque entre les structures carbonées et le milieu environnant. 


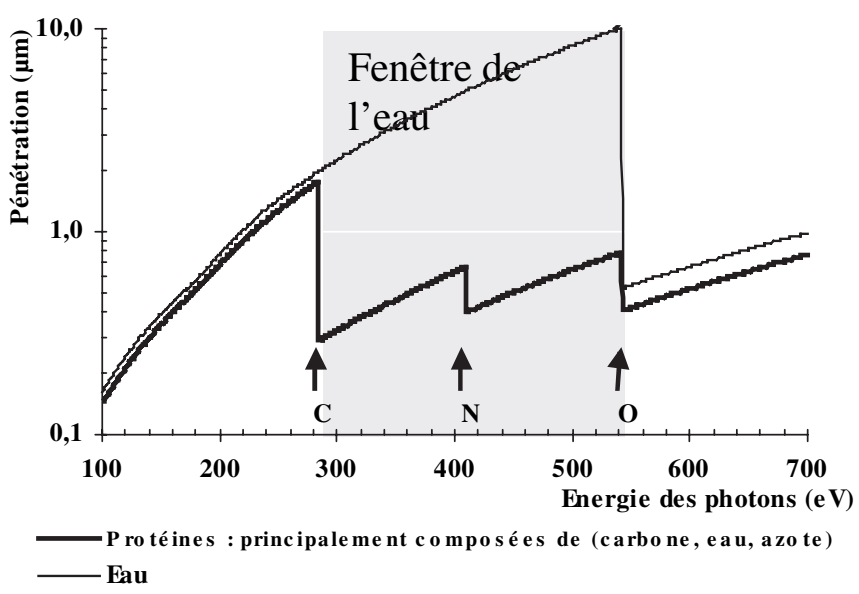

Figure 1. Pénétration comparée des rayons $\mathrm{X}$ mous dans l'eau et dans les protéines composées d'acides aminés (ici l'asparagine: $\mathrm{C}_{4} \mathrm{H}_{8} \mathrm{O}_{3} \mathrm{~N}_{2}$ ). La pénétration du rayonnement est l'épaisseur de matériau qui atténue le faisceau d'un facteur 1/e. Les seuils $\mathrm{K}$ du carbone $(284 \mathrm{eV})$ de l'azote $(410 \mathrm{eV})$ et de l'oxygène $(543 \mathrm{eV})$ sont visibles. La zone spectrale comprise entre le seuil K du carbone et le seuil K de l'oxygène est appelée "Fenêtre de l'eau ». Il existe dans cette fenêtre spectrale un contraste naturel entre les protéines et l'eau, l'eau étant 10 à 20 fois moins absorbante que les protéines pour ces énergies.

La résolution accessible en rayons $\mathrm{X}$ n'est pas la résolution ultime : la technologie actuelle des objectifs [11] empêche de s'approcher de l'ouverture numérique maximale, comme on le fait en optique. Les objectifs sont en effet des zones de Fresnel (Micro Fresnel Zone Plates, $\mu \mathrm{ZP}$ ), dont la résolution est 1.22 fois la largeur de la dernière zone.

Ces zones circulaires doivent être épaisses de $100 \mathrm{~nm}$ au moins, afin de présenter suffisamment d'absorption ou de différence de phase pour focaliser correctement le faisceau, ce qui représente un véritable défi technologique. De nos jours, les meilleures zone plates ont des dernières zones dont la largeur est comprise entre 20 et $30 \mathrm{~nm}$, permettant d'obtenir des images ayant ce type résolution latérale $[12,13]$. Des $\mu \mathrm{ZP}$ dont la dernière zone fait $10 \mathrm{~nm}$ de large sont en cours d'élaboration au Centre for $\mathrm{X}$-ray Optics (CXRO, Berkeley, Californie, USA).

La pénétration du faisceau dans l'eau est proche de $10 \mu \mathrm{m}$ à $500 \mathrm{eV}$. Cette valeur, associée à la grande profondeur de champ qui résulte de la faible ouverture numérique des $\mu \mathrm{ZP}$ permet de faire de la tomographie pour obtenir des images 3D de cellules entières avec une résolution de $60 \mathrm{~nm}$ environ [14].

L'expérience des marquages à l'or colloïdal de la microscopie électronique peut être transférée à la microscopie par rayons $\mathrm{X}$ et on peut par ce biais localiser certaines protéines avec une résolution approchant les $30 \mathrm{~nm}[12]$.

La microscopie par rayons $\mathrm{X}$ dans la fenêtre de l'eau permet donc de faire des images de cellules entières (ou de noyaux) en 2D ou 3D avec une résolution latérale de 20 à $60 \mathrm{~nm}$ selon la technologie et la modalité d'imagerie employées.

Cette technique est cependant invasive : les doses de $10^{6}$ à $10^{8}$ Gy nécessaires pour réaliser une image, provoquent des pertes de masse et des changements morphologiques instantanés sur la plupart des spécimens [15], prohibant ainsi les acquisition multiples pour la tomographie [11]. Seuls les échantillons les plus robustes comme les spermatozoïdes humains peuvent être imagés vivants sans traitement préalable et sans altérations dues à l'imagerie [16]. Des échantillons congelés rapidement sous forme de glace amorphe (Flash Freezing) à la température de l'azote liquide sont peu altérés, et peuvent supporter jusqu'à plusieurs $10^{10} \mathrm{~Gy}$ [17]. Cette technique a permis de faire de la tomographie de cellules entières congelées dans leur milieu naturel ouvrant des perspectives sur de la nanoimagerie 3D morphologique ou fonctionnelle (avec des marqueurs à l'or ou à l'argent colloïdal) de cellules $[14,18]$. 
La cryo-microscopie X, bien que moins bien résolue à l'heure actuelle que la microscopie électronique à transmission, est beaucoup plus simple d'utilisation et moins source d'artefacts.

\subsection{Problématique}

L'expérience des microscopes à rayons $\mathrm{X}$ par transmission opérant dans la fenêtre de l'eau, se situe principalement au niveau des centres de rayonnement synchrotron. Il existe trois microscopes de ce type au monde: à l'Advanced Light Source [19] (ALS, Berkeley, CA, USA) ; à Bessy II [20] (Berlin, Allemagne) et à l'Intitute for Storage Ring Facilities [21] (ISA, Aarhus, Danemark).

Ces microscopes produisent de très belles images en $2 \mathrm{D}$ (résolution $25-30 \mathrm{~nm}$ ) ou $3 \mathrm{D}$ de cellules vivantes ou congelées (résolution latérale 50-60 nm), avec ou sans marquage immunochimique [14, $16,18]$. Ces lignes de lumières sont néanmoins peu utilisées par les biologistes, car seules 2 équipes $[14,16]$ de biologistes y travaillent régulièrement, les autres expériences étant principalement menées par des physiciens [18, 20, 22, 23]. En effet, un établissement de rayonnement synchrotron n'est pas l'environnement propice pour les études de biologie cellulaire car il impose une planification à long terme et le déplacement des échantillons, souvent incompatibles avec les expériences. De plus les biologistes ne trouvent pas l'environnement qui leur est nécessaire avec les autres modalités d'observation et d'analyse. Ce manque de souplesse n'est pas adapté aux protocoles de recherche en biologie et freine la demande.

Nous proposons dans le cadre de cette étude, de rédiger le cahier des charges pour la réalisation d'un microscope à rayons $\mathrm{X}$ dit « de laboratoire », qui pourrait être placé au sein même des laboratoires de biologie, au même titre qu'un microscope électronique. L'étude a porté sur les performances optiques requises pour un tel appareil compact, afin de répondre au mieux à la demande des biologistes, mais également sur les éléments clés permettant sa réalisation : source, optiques X, détecteur, environnement de l'échantillon. Notamment, en ce qui concerne la source compacte et les optiques associées, il semble important d'exploiter les nombreux efforts réalisés dans ce domaine, pour la lithographie extrême UV. Le parallèle s'avère cependant très difficile car le cahier des charges est bien différent, de même que les performances (source et optique) que l'on peut espérer obtenir à $2.4 \mathrm{~nm}$ par rapport à $13.5 \mathrm{~nm}$.

\section{LE MICROSCOPE À RAYONS X DE LABORATOIRE}

\subsection{Description}

Un microscope à rayons $\mathrm{X}$ de laboratoire opérant dans la fenêtre de l'eau a été développé au Royal Institute of Technology de Stockholm par le Pr Hans Hertz et ses collaborateurs [24] (Figure 2).

La source est constituée d'un laser Nd:YAG, $100 \mathrm{~Hz}, 532 \mathrm{~nm}$, délivrant $100 \mathrm{~mJ}$ par impulsions de $3 \mathrm{~ns}$, focalisé sur un micro-jet d'éthanol ou de méthanol liquide (10 $\mu \mathrm{m}$ de diamètre), dans le vide [25]. De l'interaction entre le laser et la cible on obtient après filtrage des autres raies, une source sphérique, isotrope de $25 \mu \mathrm{m}$ de diamètre environ, émettant à $3.37 \mathrm{~nm}$ (raie d'émission du carbone hydrogénoïde) sur $4 \pi \mathrm{sr}$, avec une brillance de $10^{10}$ photons $/\left(\mathrm{s} \mathrm{mm}^{2} \mathrm{mrad}^{2} 0.1 \% \mathrm{BW}\right)$ et une bande passante de $\frac{\lambda}{\Delta \lambda} \geq 500$ (résolution limitée par le spectromètre).

Ce faisceau est collecté en incidence normale, par un miroir sphérique constitué de 200 bicouches de $\mathrm{W} / \mathrm{B}_{4} \mathrm{C}$ sur un substrat sphérique (diamètre : $58 \mathrm{~mm}$, rayon de courbure $343 \mathrm{~mm}$ ), placé à $260 \mathrm{~mm}$ de la source [26]. La réflectivité moyenne de ce miroir est de $0.5 \%$. Ce miroir condense le faisceau sur l'échantillon, sur une tache focale homogène d'environ $20 \mu \mathrm{m}$ de diamètre. L'objectif est une $\mu \mathrm{ZP}$ en nickel de $56.1 \mu \mathrm{m}$ de diamètre ( 468 zones, $\mathrm{f}=498 \mu \mathrm{m}$, largeur de la dernière zone : $40 \mu \mathrm{m}$ ) d'efficacité $7 \%$ à $3.37 \mathrm{~nm}$ [27]. L'image de l'échantillon est projetée sur une caméra CCD illuminée en face arrière pour une détection directe des photons X $(1024 \times 1024$ pixels, taille des pixels : $24 \mu \mathrm{m}, \mathrm{DQE}>0.6)$. En 
ajustant la distance entre la caméra CCD et l'objectif, le grossissement varie de $\times 650$ à $\times 1000$ [27]. L'échantillon est placé dans une chambre à vide et est protégé du rayonnement direct de la source par un écran central (Figure 2).

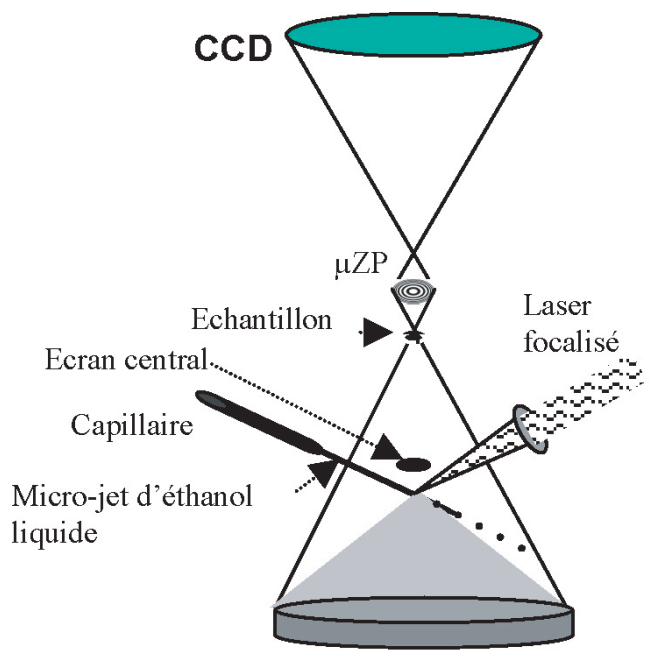

Miroir Multicouche sphérique

Figure 2. Schéma du microscope à rayons X du KTH de Stockholm. d'après [24].

\subsection{Performances}

Avec la configuration décrite au paragraphe 2.1, le détecteur collectait $3 \mathrm{ph} / \mathrm{pixel} / \mathrm{s}$ en l'absence d'échantillon, avec des optiques neuves. Au bout de deux ans d'utilisation, le flux détecté a sensiblement diminué, ce qui soulève le problème des débris de la source, qui, même s'ils sont minimisés par l'utilisation d'une source liquide sous forme de micro-jets [25], détériorent les optiques. Un flux de $3 \mathrm{ph} / \mathrm{pix} / \mathrm{sec}$ donne une image de qualité raisonnable (1000 ph/pixels) en 5 min environ, ce qui est trop long pour une utilisation de routine.

Pourtant, la source utilisée par H. Hertz a une brillance qui a le bon ordre de grandeur pour faire de la microscopie $\mathrm{X}$ de laboratoire, dans la fenêtre de l'eau, dans un temps raisonnable. Le problème principal est que la réflectivité du condenseur est très faible et inhomogène $(0.5 \%$ en moyenne avec des pics à $3 \%)$ pour des raisons d'inhomogénéités et de rugosité du substrat et des dépôts $(<0.9 \mathrm{~nm}$ d'épaisseur).

De plus, l'environnement de l'échantillon (vide) restreint les applications aux objets tests et aux cellules fixées, hors de leur milieu. De plus il existe des problèmes de vibrations. En définitive, la résolution effective est de $60 \mathrm{~nm}$ sur objets tests, et peut être réduite à $50 \mathrm{~nm}$ après traitement d'images par ondelettes [28].

\section{PERSPECTIVES}

De nombreuses optimisations sont nécessaires pour faire du microscope de laboratoire, tel qu'il est décrit par H. Hertz et ses collaborateurs dans [25], un outil utilisable en biologie cellulaire. Typiquement, il faudrait gagner deux ordres de grandeur dans la brillance de la source ou dans l'efficacité des optiques pour faire de l'imagerie 3D de cellules cryogéniques. 


\subsection{Source}

Les premiers espoirs résident tout d'abord dans les performances de la source. Tout d'abord, une source fonctionnant à $3.37 \mathrm{~nm}$ n'est pas optimale car la pénétration du rayonnement dans l'eau est faible à cette énergie. Il vaut mieux travailler aux énergies plus proches du seuil $\mathrm{K}$ de l'oxygène.

Pour les sources $\mathrm{X}$ dans la fenêtre de l'eau, à base de plasmas, il n'existe que deux éléments qui conviennent : le carbone pour sa raie hydrogénoïde $(3.37 \mathrm{~nm})$ et l'azote pour ses raies héliumoïde $(2.8 \mathrm{~nm})$ ou hydrogénoïde (2.48 nm) [29]. C'est cette dernière qui semble la plus indiquée pour la microscopie car la plus proche du seuil $\mathrm{K}$ de l'oxygène. La faisabilité et la stabilité d'une source basée sur l'interaction d'un laser YAG avec un micro jet d'azote liquide dans le vide été récemment démontré par Hertz et ses collaborateurs [30].

Une autre optimisation possible porte sur le laser lui-même. Il serait intéressant pour un tel projet de recherche de pouvoir disposer d'un laser plus puissant (typiquement des impulsions de $1 \mathrm{~J}$ en $1 \mathrm{~ns}$ ) et ayant une cadence de répétition plus grande $(\mathrm{kHz})$, Des problèmes thermiques apparaîtraient alors au niveau du jet et de la tuyère.

Un des défauts majeurs des sources à plasma est l'émission dans un angle solide $2 \pi$ ou $4 \pi$ selon la configuration. La plupart des photons sont perdus, malgré des miroirs multicouches de surface importante pour la collection. Une source directionnelle serait donc préférable, car elle s'adapterait mieux à des optiques de collection plus petites et plus efficaces. On peut citer ici l'existence d'une source basée sur le rayonnement Cherenkov émis par des électrons relativistes produits par un accélérateur médical $(10 \mathrm{MeV})$, et traversant une feuille mince de Vanadium [31]. Un faisceau monochromatique à $512 \pm 2 \mathrm{eV}$ est émis dans un cône ayant un $1 / 2$ angle d'ouverture de $4^{\circ}$, ce qui permettrait d'utiliser un condenseur basé sur des zones de Fresnel, ou un miroir multicouche à incidence rasante [31].

\subsection{Optique}

On peut espérer également un gros gain sur l'efficacité des optiques utilisées. On vient de voir précédemment qu'une source émettant dans un cône d'ouverture raisonnable permettrait d'utiliser des optiques plus performantes. On peut ainsi envisager l'utilisation de zone plates comme condenseurs (efficacité $\sim 10 \%$ ) ou des miroirs multicouches en incidence rasante (efficacité théorique $\sim 40 \%$ ).

De plus, il existe un grand fossé entre les optiques à multicouches utilisées en incidence normale pour la lithographie (efficacité $\sim 70 \%$ ) et celles utilisées pour les $\mathrm{X}$ mous (efficacité théorique $\sim 10 \%$ ). Là encore des progrès sont possibles à la fois dans les matériaux utilisés et dans les homogénéités des dépôts, permettant de gagner un facteur 10 dans les performances globales du système. Par exemple, à $3.37 \mathrm{~nm}$, il est recommandé d'utiliser un miroir au $\mathrm{Cr} / \mathrm{Sc}$, qui théoriquement devrait donner une réflectivité moyenne proche de $15 \%$ [32]. Un multicouche au Ni/V donnerait quant à lui une réflectivité de $2 \%$ en incidence normale à $500 \mathrm{eV}(2.48 \mathrm{~nm})$ et supérieure à $10 \%$ à $512 \mathrm{eV}$ $(2.42 \mathrm{~nm})$, grâce à la valeur élevée de l'indice de réfraction au voisinage immédiat du seuil $\mathrm{L}$ du vanadium.

Parallèlement, les progrès dans les $\mu \mathrm{ZP}$ pourront accroître la résolution limite possible en microscopie X. La réalisation de zones larges d'une dizaine de $\mathrm{nm}$ et épaisses de plus de $100 \mathrm{~nm}$ est le point critique.

\section{CONCLUSIONS}

La construction d'un microscope $\mathrm{X}$, fonctionnant dans la fenêtre de l'eau et répondant aux besoins des biologistes (imagerie 3D, résolution $<20 \mathrm{~nm}$ ) paraît techniquement faisable. Il est nécessaire pour cela de réaliser des progrès technologiques à la fois sur la source et sur l'optique, afin de gagner le facteur 100 qui manque sur l'efficacité globale du système, pour pouvoir faire une acquisition d'une cellule congelée, avec 1000 photons par pixels, en quelques secondes. 
La possibilité de réaliser des images 3D de cellules entières avec une résolution 50-100 nm sans autre préparation que la congélation est l'apport que l'on attend de cette technique.

Le coût d'un tel appareil serait comparable à celui d'un grand microscope électronique à transmission.

Il reste une incertitude sur l'intérêt d'un tel appareil pour la communauté des biologistes qui ne peut être levée : En effet, il est difficile d'évaluer si la demande reste contenue à l'heure actuelle à cause d'une méconnaissance de la technique, d'un manque d'information et de formation et des limitations intrinsèques à l'utilisation du rayonnement synchrotron, ou bien si l'apport de la microscopie dans la fenêtre de l'eau serait insuffisant pour en justifier le développement.

Il faut donc faire un pari sur l'utilisation probable de cet appareil et lancer la boucle « utilisation, développement, utilisation accrue » tout en formant et en informant la communauté des biologistes sur cette technique prometteuse.

\section{Remerciements}

Nous tenons tout particulièrement à remercier les personnes, nombreuses, qui nous ont aidés et formés aux technologies actuelles utilisées en microscopie à rayons X et aux applications biologiques associées. Merci à: J. Susini (ESRF), JP. Geindre \& C. Chenais-Popovics (LULI), M. Makarov, M.\& JL. Stehle (SOPRA SA), JM. Pouvesle, C. Cachoncinlle \& E. Robert (GREMI), Ph. Zeitoun (LOA), M. Schmidt, Ph. Martin (CEA/DRECAM); R. Lebert, W. Neff et leur équipe (AIXUV, ILT/Fraunhofer); J. Verhoeven (FOM), OJ. Luiten, W. Knulst \& MJ. Van der Viel (TUE), C. Larabell (ALS), J. Abraham-Peskir (ISA), O. Valiron (CEA/DSV), M. Robert-Nicoud (UJF), J. Conway (IBS); H. Hertz, A. Holmberg \& H. Stollberg (KTH, Stockholm), F. Bijkerk et son équipe (FOM) ; O. Renault (CEA/LETI) ; JY. Robic et son équipe (CEA/LETI) ; A. Glière (CEA/LETI) ainsi que tous les membres du projet Nanimax (F. Mathy, JM. Dinten, JL. Amans).

Nous avons une pensée particulière pour notre collègue Christine Robert-Coutant qui avait démarré ce travail, et est décédée prématurément.

\section{Bibliographie}

[1] C. Dobell. Antony van Leeuwenhoek and his "Little animals". (Dover Publications, New York, 1960)

[2] D.M. Phillips. Methods Cell Biol 57 (1998) 297-311

[3] A. Lewis, A. Radko, N. Ben Ami, D. Palanker and K. Lieberman. Trends Cell Biol 2 (1999) 70-73

[4] S. Bartolomé, A. Bermúdez and J.R. Daban. J Cell Sci 107 (1994) 2983-2992

[5] B.M. Gumbiner Cell 84 (1996) 345-57

[6] O.J. Castejón, H.V. Castejón, P. Sims J Submicrosc Cytol Pathol 33 (2001) 23-32

[7] G. Wastiaux, La microscopie optique moderne. (Lavoisier, Paris, 1994)

[8] S.J. Wright, D.J. Wright. Methods Cell Biol 70 (2002) 1-85

[9] J.L. Alonso, W.H. Goldmann. Life Sci 72 (2003) 2553-2560

[10] S.W. Hell Nat Biotechnol 21 (2003) 1347-1355

[11] C. Jacobsen Trends Cell Biol 9 (1999) 44-47

[12] W. Meyer-Ilse, D. Hamamoto, A. Nair, S.A. Lelievre, G. Denbeaux, L. Johnson, A.L. Pearson, D. Yager, M.A. Legros and C.A. Larabell. J Microsc Oxford 201 (2001) 395-403

[13] W.L. Chao, E. Anderson, G.P. Denbeaux, B. Harteneck, J.A. Liddle, D.L. Olynick, A.L. Pearson, F. Salmassi, C.Y. Song and D.T. Attwood. Opt Lett. 28 (2003) 2019-2021

[14] C.A. Larabell and M.A. Le Gros. Mol Biol Cell. 15 (2004) 957-962

[15] K. Shinohara and A. Ito. J Microsc Oxford 161 (1991) 463-472

[16] J.V. Abraham-Peskir, E. Chantler, E. Uggerhøj and J. Fedder Hum Reprod 16 (2002) 375-382

[17] G. Schneider. Ultramicroscopy 75 (1998) 85-104

[18] G. Schneider, E. Anderson, S. Vogt, C. Knochel, D. Weiss, M. Legros, and C. Larabell. Surf Rev Lett 9 (2002) 177-183 
[19] W. Meyer-Ilse, H. Medecki, L. Jochum, E. Anderson, D. Attwood, C. Magowan, R. Balhorn, M. Moronne, D. Rudolph and G. Schmahl. Synchrotron Radiation News 8 (1995) 29-33

[20] P. Guttmann, B. Niemann, J. Thieme, U. Wiesemann, D. Rudolph and G. Schmahl in X-ray Microscopy, Proceedings of the VI International conference (Aip Conference Proceedings vol 507), Berkeley, 1996, edited by W. Meyer-Ilse, T. Warwick, D. Attwood (American Institute Of Physics, New york, 2000), pp 411-415

[21] R. Medenwaldt and E. Uggerhøj. Rev Sci instrum 69 (1998) 2974-2977

[22] J. Wendt-Larsen, J.V. Abraham Peskir, R. Medenwaldt Cell Biol Int 25 (2001) 521-530

[23] E.G. Søgaard, R. Medenwaldt, J.V. Abraham Peskir Wat Res 34 (2000) 2675-2682

[24] G.A. Johansson, A. Holmberg, H. Hertz and M. Berglund Rev. Sci. Instrum. 73 (2002) 1193-1197

[25] J. de Groot, O. Hemberg, A. Holmberg, H.M. Hertz J Appl Phys 94 (2003) 3717-3721

[26] H.M. Hertz, L. Rymell, M. Berglund, G. A. Johansson, T. Wilhein, Y. Platonov and D. Broadway, SPIE proceedings 3766 (1999) 247-251

[27] M. Berglund, L. Rymell, M. Peuker, T. Wilhein and H.M. Hertz J Microsc Oxford 197 (2000) 268-273

[28] H. Stollberg, J. Boutet de Monvel, A. Holmberg and H.M. Hertz J Microsc Oxford 211 (2003) 154-160

[29] R. Lebert, W. Neff, D. Rothweiler. Journal of X-Ray Science and Technology 6 (1996) 107-140

[30] H. Hertz, G.A. Johansson, H. Stollberg, J. de Groot, O. Hemberg, A. Holmberg, S. Rehbein, P. Jansson, F. Eriksson \& J. Birch. J. Phys. IV 104 (2003) 115-119

[31] W. Knulst, M.J. van der Wiel, O.J. Luiten and J. Verhoeven Appl Phys Lett 83 (2003) 4050-4052

[32] F. Eriksson, G.A. Johansson, H.M. Hertz, E.M. Gullikson, U. Kreissing \& J. Birch. Opt. Lett. 28, (2003) 2494-2496 